\title{
Res publica revisited
}

Mit diesem Doppelheft der Zeitschrift für Staats- und Europawissenschaften (ZSE) summieren Herausgeber wie weitere Kollegen der Rechts-, Wirtschaftsund Sozialwissenschaften den Stand der Staatsdiskussion, die in den vergangenen Jahren - zunächst eher unbeachtet, dann umso intensiver - einer Reihe situativ wie strukturell bedeutsamer Auseinandersetzungen unterlag. Waren es zunächst Formen außerstaatlichen oder überstaatlichen Handelns, die eine Überprüfung des tradierten Staatsverständnisses nahe legten, traten im Rahmen der aktuellen Krisenbewältigung und mit der Entscheidung des Bundesverfassungsgerichts zum Vertrag von Lissabon weitere Themenfelder hinzu. Im Ergebnis sind es die damit verbundenen endogenen wie exogenen Herausforderungen des staatlichen Handelns, die die Begründung für dieses Doppelheft bilden. Es geht also um wissenschaftsimmanente wie extern induzierte Veränderungen, die einer näheren Bestandsaufnahme und Kommentierung bedürfen. Res publica revisited bildet die Metapher für dieses Unterfangen.

Die damit angesprochenen Veränderungen der staatlichen Rolle und Funktion finden sich auf unterschiedlichen Ebenen. Während die Feierlichkeiten zum 60jährigen Bestehen des Grundgesetzes auf eine auch im internationalen Vergleich bemerkenswerte Stabilität und Leistungsfähigkeit der Verfassung verwiesen, stehen daneben zahlreiche Diskussionen, die grundlegender verfassungstheoretischer wie verfassungspolitischer Natur sind und sich vor allem auf die Wirkungsweise der staatlichen Organe und Einrichtungen (auf allen gebietskörperschaftlichen Ebenen) richten. Dies reicht von einer Überprüfung des institutionell-organisatorischen Rahmens, etwa der bundesstaatlichen Ordnung durch die beiden Föderalismuskommissionen, bis hin zu dem Versuch, über eine den Namen verdienende Aufgabenkritik und Prozessreform das öffentliche Handeln problemadäquater, zukunftsorientierter und nachhaltiger zu gestalten. Da „regulative Politiken“ zudem als nur noch bedingt wirkungsmächtig gelten, tritt ein Blick auf verfahrensbezogene und instrumentelle Fragen hinzu. Hier geht es um die Überprüfung des staatlichen Steuerungsanspruchs und etwaiger „Schnittstellen“ zum privaten Bereich, sucht eine Führungsdiskussion die materielle Kompetenz der politisch Handelnden zu verbessern und zwingt die desola- 
te Haushaltssituation zu einem konsequenteren Umgang mit knapper werdenden (finanziellen wie personellen) Ressourcen. Im Ergebnis sehen zahlreiche Beobachter in der Trias Wirtschaft- und Finanzkrise, zunehmende interne wie externe Anforderungen und eingeschränkte Leistungsfähigkeit der öffentlichen Einrichtungen eine nicht nur auf Diskussion, sondern auch auf Reformen drängende Ausgangssituation. Dies mag erklären, warum in Ergänzung dauerhafter Erörterungen etwa des Verhältnisses von Nationalstaat und Europäischer Union oder eines zeitgemäßen Regierungs- und Verwaltungshandelns inzwischen auch grundlegendere Fragen zur Reformfähigkeit demokratischer politischer Systeme gestellt werden, die Untersuchungen zur Handlungsbereitschaft, Handlungsfähigkeit und Bindungswirkung im Rahmen etablierter Verfahren mit Fragen nach äquifunktionalen Mechanismen verbinden.

Wie meist, so sind auch diese Diskussionen durch eine beträchtliche Überschussenergie gekennzeichnet, die in dem Anwachsen untypischer Netzwerke und neuerer akteursspezifischer Konstellationen den Übergang nicht nur in eine neue Qualität, sondern auch in eine neue Form öffentlichen Handelns zu sehen glauben - bis hin zu eher modischen Varianten, die angesichts grenzüberschreitender Probleme bereits das Ende des Nationalstaates auszurufen suchen. Dabei wird schon aus historischer Sicht übersehen, dass der deutsche Staat in einer durchaus guten (und ausbaubaren) Tradition steht. Sie ermöglichte 1919 den Übergang von der Monarchie in die Republik und war - obwohl sie 1933 den Weg vom Rechts- in den Unrechtsstaat nicht zu verhindern vermochte - 1945 doch noch so greifbar, dass man in Teilen an sie anknüpfen konnte, als zunächst in Gemeinden und Kreisen und etwas später in den Ländern das öffentliche Leben wieder seinen Anfang nahm. Sieht man dann von dem ab, was in der DDR geschah, hat die Entwicklung in der alten Bundesrepublik auf der einen Seite zu einer Bestätigung und Ausweitung positiver Staatstraditionen beigetragen. Dies gilt nicht zuletzt für das in der Verfassung angelegte System von checks and balances zwischen den wesentlichen politischen Organen, für die Machtteilung zwischen den Gliedstaaten, für die Schaffung politisch unabhängiger Einrichtungen wie der Bundesbank und des Bundesverfassungsgerichts, die gleichsam korporatistische Willensbildung zwischen Arbeitgebern und Arbeitnehmern sowie für zahlreiche rechts- wie sozialstaatliche Sicherungen. Auf der anderen Seite kam es aber auch zu einem Qualitätsverlust von Staat und Verwaltung insofern, als sich eine politische Willensbildung herausbildete, die weit über das vom Grundgesetz geforderte Maß hinaus ging: eine zunehmende Verflechtung zwischen den gebietskörperschaftlichen Ebenen, die Forderungen nach politi- 
scher Rationalität und aktiver bürgerschaftlicher Beteiligung entgegenstand; ein Anwachsen der Verwaltung, die heute durch deutliche horizontale wie vertikale Koordinationsprobleme gekennzeichnet ist und kaum mehr bezahlbar erscheint; eine Spezialisierung, die übergreifende Problemlösungen erschwert; eine Politisierung öffentlicher Einrichtungen mit sich damit verbindenden Einbußen an rechtstaatlicher Qualität sowie schließlich ein erkennbares Unvermögen, die Frage, wie viel Staat wir brauchen, auch wirklich zu stellen und sie handlungsleitend zu beantworten.

Fasst man den heutigen Stand der staatswissenschaftichen wie staatspraktischen Diskussion zusammen, ergibt sich mit Blick auf aktuelle wie strukturelle Herausforderungen zunächst ein weitgehender Konsens: Danach gelten der Wohlfahrtsund Sozialstaat der zweiten Hälfte des 20. Jahrhunderts aufgrund des inzwischen wesentlich ausdifferenzierteren Parteienwettbewerbs, des ökonomischen Strukturwandelns, der offensichtlichen sozialen Kosten und eines überholten staatlichen Paradigmas (Staat als omnipotente autonome Steuerungsinstanz einer Gesellschaft) als Ausdruck einer eher historischen Zwischenphase. So haben sich die Staatsfunktionen zunehmend vom Leistungs- zum Steuerungs- und Ordnungsstaat verschoben, im Sinne der Organisation gesellschaftlicher Interaktions-, Produktions- und Entscheidungsprozesse. Dabei verlagern sich die Leistungsfunktionen zu einem nicht unbeträchtlichen Teil auf dezentrale politischadministrative Ebenen, die das Leistungsangebot den sich differenzierenden Ansprüchen präziser anzupassen vermögen, die Nachfrage wirksamer in die Angebotsgestaltung einbeziehen und besser als zentralstaatliche Einrichtungen in der Lage sind, die erforderlichen Akzeptanz- und Konsensbildungsprozesse zu gestalten. Die Autonomie des Staates, obwohl faktisch nie gegeben, wenngleich von den Handlungsträgern subjektiv so empfunden, wird immer weiter zugunsten kooperativer Strukturen zwischen Staat und gesellschaftlichen Handlungsträgern aufgelöst; die Aufgaben des Staates konzentrieren sich danach auf die Wahrnehmung von Führungsfunktionen: Kooperation, Koordination und Moderation rücken in den Vordergrund. Der Kooperationsbedarf entspringt hier durchaus staatlichen Eigeninteressen: zur Sicherung der finanziellen Voraussetzungen seines Handelns, zur Abdeckung staatlicher Aufgaben, zur Verhinderung von Legitimationsverlusten und zur Verbesserung des politischen Vollzugs. Die gesellschaftliche Komplexität, die unübersehbare Optionenmehrung individueller Interessendurchsetzung und die Unsicherheit über Wirkungsverläufe gesellschaftlicher Großentscheidungen erhöhen ständig die individuellen Risiken. Der Staat wird dabei immer häufiger in die Rolle des „,Großversicherers “ gedrängt, 
der allerdings nicht (nur) nachträglich Schäden beseitigen, sondern sie präventiv verhindern soll. Das freilich setzt vorausschauende Planung, Risikokalkulation und neue Verfahren der Entscheidungsvorbereitung voraus, die ohne gesellschaftliche Übereinstimmung nicht konsensfähig werden. Letztere aber wird bei zunehmender gesellschaftlicher Desintegration, dezentraler Steuerung und gruppengebundenen „Weltbildern“ - immer mehr zu einem knappen Gut. Die Konsensbildungskosten wachsen progressiv, weil jeweils problembezogene Übereinstimmungen hergestellt werden müssen, ein differenzierter Grundkonsens also fehlt. Aufgabe des Staates wird es dann, die Kosten der Konsensbildung zu senken, sei es durch neue Organisationsformen im Bereich der Willensbildung und Entscheidung, sei es durch Erneuerung eines entsprechend differenzierten Konsenses.

Während man gegen Ende des 20. Jahrhunderts dem mit vergleichsweise idealtypischen Vorstellungen gesamthafter Politikbearbeitung zu begegnen suchte (und dabei an institutionellen wie politischen Widerständen scheiterte), entstanden zwischenzeitlich Gegenpositionen, die stärker auf eine Selbststeuerung sozialer Systeme setzten, sei es über den Markt (Entstaatlichung, Privatisierung, Deregulierung), sei es über soziale Netzwerke (Dezentralisierung, genossenschaftliche Selbsthilfe) oder über Selbstdisziplinierungen im ,wohlverstandenen Eigeninteresse", etwa durch Anspruchsreduktion, Selbstkontrolle von Gruppen oder diskursiven Konsens. Diese Positionen gingen jedoch meist von einem insofern illusorischen Gesellschafts- und Politikverständnis aus, als sie gesellschaftliche Rationalitätssteigerung empfahlen, ohne die damit wachsenden Kosten der Konsensfindung zu berücksichtigen, integrierte Aufgabenplanung durch einen Abbau politisch-administrativer Arbeitsteilung anstrebten, ohne die informatorischen und konsensbezogenen Vorteile der Arbeitsteilung in Rechnung zu stellen, und schließlich dezentralisierte Selbststeuerung propagierten, ohne zu erkennen, dass ausdifferenzierte Gesellschaften und pluralistisch-fragmentierte Institutionensysteme einen wachsenden Kollektivbedarf an Steuerung, Planung und Konsensbildung erzeugen.

Heute kommt es daher verstärkt zu pragmatischen Ansätzen, die aufgaben- oder politikfeldbezogen vorgehen und versuchen, die einer „rationalen“ Politikbearbeitung entgegenstehenden Widerstände (aus vertikal wie horizontal verflochtenen institutionalisierten Zuständigkeiten, der Heterogenität und Vielfalt privater wie öffentlicher Entscheidungsträger oder aus materiellen Verteilungskonflikten über Ressourcen, Kompetenzen und andere Handlungsvorteile) konstruktiv auf- 
zugreifen und zu nutzen. Staatliche Politik ist dabei als Führungsaufgabe zu verstehen, der drei gleichsam ,strategische“ Funktionen zuzuordnen sind:

- eine Orientierungsfunktion zur Bestimmung und Definition von Problemen, zur Festlegung von „Fluchtlinien“ des Handelns und zur Präzisierung der erwarteten (und nachprüfbaren) Handlungsergebnisse;

- eine Organisationsfunktion, die sicherstellen muss, dass alle wichtigen Handlungsträger für ein Politikfeld mobilisiert und zu gemeinsamer Handlung zusammengeführt werden; der Staat/die öffentliche Hand kann immer weniger alle Probleme einer Gesellschaft an sich ziehen und aus eigener Kraft zu lösen versuchen, da die Problemfelder nur zu einem kleinen Teil von Variablen gebildet werden, auf die er/sie direkt Einfluss nehmen kann; darüber hinaus verfügen Private über ein zunehmend wachsendes Störpotential, das sie - auch ungewollt, etwa durch egoistisches Verhalten - gegen den Staat einsetzen; und schließlich

- eine Vermittlungsfunktion, die es zur Aufgabe hat, Konsens und Akzeptanz für gemeinsame Handlungswege zu schaffen und die verschiedenen Handlungsträger zu motivieren.

Die Orientierungsfunktion geht davon aus, dass die Arbeitsteilung einer Gesellschaft und die Erkenntnis, dass in komplexen Kontexten Entscheidungen/Handlungen lange Wirkungsketten auslösen, deren Ergebnisse häufig Folgeprobleme schaffen, den Staat immer mehr in Steuerungsfunktionen gedrängt haben, die eine Vielzahl von Adressaten beeinflussen müssen. Orientierung geben heißt dann: Probleme frühzeitig aufzugreifen und zu benennen, Optionen für die Problembearbeitung zu schaffen, Ziele und Leitlinien für Problemlösungen zu bezeichnen und Handlungen daraufhin abzuschätzen, welche gewollten und ungewollten Folgewirkungen sie haben (,Aktivitätsfolgenabschätzung“: Umweltverträglichkeitsprüfung, Technologiefolgenabschätzung, Sozialverträglichkeitsprüfung, Raumverträglichkeitsprüfung etc.). Orientierung ist aber nicht nur handlungstrategisch zu verstehen, sondern wirkt auch auf die Grundwerte einer Gesellschaft ein, die letztlich den Möglichkeitsrahmen des Handelns mitbestimmen, aber auch - wie im Fall von Solidarität - die Voraussetzungen für kollektives Handeln darstellen.

Die Organisationsfunktion stellt gleichsam das materielle Korrelat zur Orientierungsfunktion dar. Sie unterscheidet die Organisation von Interaktionsprozessen zur Konsensfindung und Akzeptanzgewinnung sowie die Organisation der Kompetenz- und Ressourcenbereitstellung, um kollektives Handeln materiell erst zu ermöglichen. Die interaktionsbezogene Organisationsfunktion kann dabei politische Handlungssysteme zum Gegenstand haben (etwa konzertierte Aktionen 
oder Regionalkonferenzen) oder ordnungspolitische Regelungen schaffen, die wie Marktordnungen - das Handeln Dritter strukturell gestalten.

Die Vermittlungsfunktion verweist darauf, dass kollektives Handeln nicht nur initiiert, sondern auch motiviert und moderiert werden muss, zumal der Staat immer stärker auf die Mitwirkung privater und nicht-staatlicher Organisationen angewiesen ist, denen gegenüber Ge- und Verbote häufig wirkungslos bleiben, weil sie von den Adressaten unterlaufen bzw. von den ausführenden Behörden nicht adäquat umgesetzt werden (können). Vermittlung besteht dann nicht nur in der Verkündung von Zielen, Programmen oder in der Gewinnung von Kooperationspartnern, es geht vielmehr auch um den Einbezug derer, die an der kollektiven Aktion beteiligt sind - und dies bereits bei der Entwicklung und Planung von Zielen wie Maßnahmen. Die Aufgabe ist also sowohl intern (bezogen auf die öffentlichen Handlungsträger) als auch extern (bezogen auf nicht-staatliche Handlungsträger) zu verwirklichen. Sie kann umgesetzt werden über Beratungsleistungen, Überzeugungsarbeit, Tauschhandlungen und Verhandlungsprozesse. Die Wahrnehmung der Vermittlungsfunktion geht dabei häufig über den Informationstausch hinaus, meist handelt es sich auch um einen Prozess politischer Konfliktregelung, der wiederum Rückwirkungen auf die Organisationsfunktion haben kann.

Die Wahrnehmung aller drei genannten Funktionen, die den Staat zumindest auch als ,kooperativen“ Staat ausweisen, könnte dazu beitragen, Probleme früher aufzugreifen und damit den Staat wieder zum „Herrn des Verfahrens", in freilich verändertem Verständnis, zu machen. Dies bezieht sich sowohl auf den Zeitpunkt der Problembearbeitung, deren zieladäquate und ressourcenschonende Gestaltung sowie schließlich auch auf die Schnelligkeit und die Dauerhaftigkeit von Problemlösungen.

Die Entscheidung für die „öffentliche Hand“, den Staat (in seinen unterschiedlichen Erscheinungsformen) ist also nach all dem selbstverständlich. Sie erklärt allerdings noch nicht, welcher Staat gemeint ist und was man ihm im Einzelnen abfordern sollte. So gilt es den angesprochenen Wandel und die Veränderung der gesellschaftlichen Rahmenbedingungen zu berücksichtigen, der zunehmenden territorialen wie sektoralen „Entgrenzung“ einstmals selbstverständlicher Grundlagen $\mathrm{zu}$ folgen und dabei normativen wie funktionalen Herausforderungen zu begegnen. Was man dabei vom Staat, seiner Verfassung und der in ihrem Rahmen geschaffenen Ordnung erwarten kann, ist, dass sie so stabil und dauerhaft wie möglich und so flexibel wie nötig sind. Der von manchen beklagte Verfassungswandel gehört so in Wahrheit zur Normalität moderner „Staatlichkeit“. 
Angesichts dieser Ausgangssituation könnte man erwarten, dass sich die den Staatswissenschaften zugewandten Disziplinen auf die in der Praxis erkennbaren Veränderungen der Aufgaben-, Organisations-, Entscheidungs- und Ressourcenstrukturen konzentrieren, historisch wie theoretisch angeleitet und empirischanalytisch grundiert. Stattdessen kam es zu Interpretationsansätzen, die höchst ausdifferenzierten Problemen und Prozessen nur eher gesamthaft zu entsprechen und sich den angesprochenen endogen wie exogen induzierten Veränderungen eher über neue Begrifflichkeiten zu nähern suchten. Dies galt zwischenzeitlich etwa für den ubiquitären Einsatz des Begriffs governance, der sich (wie einige seiner Vorgänger) freilich rasch als analytisch unterkomplex erwies. Meist ohne definiertes Subjekt und Objekt, frei von Zeit-Raum-Differenzierungen und kaum zwischen abhängigen, unabhängigen und intervenierenden Variablen unterscheidend, wird die Chiffre heute eher von Öffentlichrechtlern eingesetzt, die sich aus der stringenten Logik ihrer Disziplin verabschiedet haben, oder aber von Sozialwissenschaftlern, die über breite Begrifflichkeiten einem differenzierteren $\mathrm{Zu}$ gang und einer anspruchsvolleren Empirie auszuweichen suchen. So wurde die Bezeichnung, wie viele andere vor ihr, letztlich zu einer fast beliebigen (catch all-) Kategorie, die eher „vernebelte“ denn klärte, suchte man über die englischsprachige Begrifflichkeit den Anschluss an eine Diskussion, die seit wenigstens vierzig Jahren im US-amerikanischen Kontext geführt wird. Ein Blick auf die solchen Fragen seit langem gewidmete Zeitschrift (im Wiley-Verlag) macht die Anleihen deutlich und reduziert jenes Alleinstellungs- oder gar Innovationsmerkmal, das man deutscherseits für sich in Anspruch zu nehmen suchte.

An diesem Beispiel wird auch deutlich, dass und wie schnell Teile der die Staatswissenschaft konstituierenden Disziplinen gelegentlich ihre Identität aufzugeben bereit sind, samt der damit drohenden Traditionslosigkeit, Kontinuitätsbrüche und wachsenden Distanz zum Gegenstandsbereich. Gerade in Themenfeldern, die wie wenige andere auf Interdisziplinarität, internationalen Vergleich und Interkulturalität angewiesen sind, sollten Prozesse der Selbstisolation vermieden und der Einsatz dessen selbstverständlich sein, was sich analytisch bewährt hat: vom historischen wie akteursspezifischen Institutionalismus über Interorganisationsanalysen bis hin zu Formen und Funktionen regulativen Handelns. Ergänzt um das inzwischen reichhaltige Arsenal der policy-Analytiker und den Ertrag der empirischen Sozialforschung ergibt sich so eine Basis seriöser Staatswissenschaft, die über den Einbezug territorialer, sektoraler oder auch sozialkultureller Entgrenzungsprozesse längst die Fixierung auf den Nationalstaat überwunden hat, ohne sich von ihm als dem zentralen Akteur zu verabschieden. Nicht nur 
Ralf Dahrendorf wies kontinuierlich darauf hin, dass es kein Zufall ist, wenn Länder, in denen moderne Bürgerrechte sich erst später durchgesetzt haben, meist auch verspätete Nationen waren, während die ersten Nationen zugleich Vorreiter der Bürgerrechte wurden. Der moderne Nationalstaat stellt im Kern die Form dar, in der das nicht-feudale, auch anti-feudale Bürgertum seinen Platz finden konnte. Das Bürgertum brauchte die Nation, um Recht und Verfassung an die Stelle von überlieferten Bindungen und Gottesgnadentum zu setzen. Insoweit ist der Nationalstaat Quelle des Fortschritts auf dem Weg zu einer allgemein das Recht verwaltenden bürgerlichen Gesellschaft. Dies schließt nicht aus, dass dem Nationalstaat von Anfang an ein Janusgesicht beigegeben wurde. Er hat einerseits alte Standesgrenzen überwunden und andererseits durchaus neue Grenzen geschaffen. Noch heute schließt der Nationalstaat ebenso aus wie ein. Doch bleibt auch unübersehbar, dass das Bündnis von Liberalismus und Nationalismus nicht nur während der evolutionären Jahrzehnte von 1789 bis 1848 eine Kraft der Emanzipation bildete. Bis heute hat niemand eine effektivere Garantie des Rechtsstaates, seiner Verfassung der kontrollierten Macht, seiner verlässlichen Verfahrensregeln und regelmäßigen Entscheidungskontrollen gefunden. Nicht der geringste Vorzug des Nationalstaates war es zudem, dass er es erlaubte, die Idee der Bürgerrechte zu verallgemeinern.

\section{Zum Verhältnis von Nationalstaat und Europäischer Union}

Das Verhältnis von Nationalstaat und Europäischer Union (oder anderen Formen überstaatlicher Regionalorganisation) ist mithin von zentraler Bedeutung bei der Bewältigung der in diesem Heft angesprochenen Herausforderungen und bildet einen der drei Schwerpunkte, um den herum sich die nachfolgenden Beiträge gruppieren. „Lissabon und die Folgen“ (I.) wurde dabei als Überschrift gewählt, weil sich im Rahmen dieser Entwicklung exemplarische Veränderungen im Verhältnis von Nationalstaat und Europäischer Union aufzeigen lassen. Ohne hier die Erörterungen der viel diskutierten „Lissabon-Entscheidung“ des Bundesverfassungsgerichts vorwegzunehmen, sei daran erinnert, dass das Gericht den Bundestag aufforderte, bei einer Übertragung weiterer Kompetenzen auf überstaatliche Einrichtungen seiner „Integrationsverantwortung“ gerecht zu werden. Die sich an dieses Urteil anschließende Diskussion spaltete dann die einflussreiche Gruppe der deutschen Staatsrechtslehrer, von denen die einen gleichsam kampagnenartig den Zweiten Senat und hier vor allem den Berichterstatter einer gleichsam „vormodernen“ Sichtweise des Europäisierungsprozesses bezichtigten, während andere die demokratietheoretisch und demokratiepolitisch 
grundierte Argumentation des Zweiten Senats begrüßten und dies mit der Empfehlung verbanden, künftig dem fortschreitenden europäischen Integrationsprozess in einer pragmatischeren, funktional wie politisch angemesseneren und professionelleren Haltung zu begegnen. Während dabei erkennbar wurde, dass vor allem Europarechtler, unter Beifall der um ihre Definitionsmacht besorgten Politik, ihre disziplinäre Position zu verteidigen suchten und die Prägekraft des Europarechts wie des Europäischen Gerichtshofs betonten, verwies die zweite Gruppierung auf funktionale wie legitimatorische Fehlentwicklungen, die es, erst recht in der Kontinuität der europabezogenen Rechtsprechung des Bundesverfassungsgerichts, angeraten erscheinen ließen, den im Zeitablauf entwickelten „Grenzziehungen“ deutlicher und vor allem konsequenter als bislang zu folgen.

Die über Aufsätze und Leserbriefe vor allem in der Frankfurter Allgemeinen Zeitung gleichsam öffentliche Diskussion kulminierte schließlich unter Einsatz von Invektiven wie ,ausbrechender Rechtsakt“ oder „Staatsverständnis des 19. Jahrhunderts" in dem Bemühen, das Bundesverfassungsgericht gleichsam der Gestrigkeit zu überführen, unter Einschluss von Empfehlungen, den Auswahlprozess künftiger Richter zu überprüfen. Dabei vergaß man im Kreis der als gleichsam „Berufseuropäer“ Agierenden, dass der Integrationsprozess bislang keineswegs bruchlos verlief und es seit geraumer Zeit durchaus nahe liegt, eine Konsolidierungs- und Stabilisierungsphase vorzusehen, die anstelle inzwischen entbehrlicher „Überzeugungspolitiken“ ein verbessertes operatives Handeln vorsieht und vor allem eine kontinuierliche „Rückbindung“ der europäischen Einrichtungen gewährleistet. Auch sei daran erinnert, dass die ambitionierten Pläne zur Schaffung einer Europäischen Verfassung ja nicht eben grundlos scheiterten, da man nicht nur vergaß, die „Völker Europas“ in diesem Prozess mitzunehmen, sondern auch glaubte, in einem eher technokratischen Akt den Europäern eine Verfassung vorgeben zu können. Dies scheiterte an eben diesen „Völkern“, zunächst in Gestalt der Franzosen und Niederländer, später der Iren. Die arroganten Reaktionen, die auf diese Referenden folgten, richteten sich schließlich selbst. Gleichwohl fanden sie auch in die Auseinandersetzungen um das Urteil des Bundesverfassungsgerichts Eingang, bis ein sehr deutlicher Leserbrief des ehemaligen Bundestagsvizepräsidenten Burkhard Hirsch die Diskussionen zumindest vorläufig beendete:

Der Autor (gemeint ist Carl Otto Lenz) setzt die unselige Tradition insbesondere westdeutscher Europa-Politiker fort, jeden kritischen Gedanken zu Art und Inhalt des europäischen Einigungsprozesses als „nationalistisch“, jedenfalls schädigend und unhistorisch zu disqualifizieren. Ausgerechnet an uns dürfte die europäische Integra- 
tion nicht scheitern, ist die gängige Argumentation. Als ob es besser wäre, wenn sie ausgerechnet an Frankreich, Irland, Großbritannien oder Italien scheitern würde.

Ein Gespenst geht um in Europa: das Volk. Europa steht auf schwachen Füßen, wenn es zum Tabu wird, das man vor offenen Diskussionen und vor einem Referendum bewahren muss. Sind wir dümmer als andere Völker, oder trauen wir es nur unseren Politikern nicht zu, dem Wähler Europa zu erklären? Und war es nicht ein geradezu empörender Versuch, uns mit der heuchlerischen Bezeichnung „Verfassungsvertrag" und dann mit wenigen kosmetischen Reparaturen eine Verfassung zu oktroyieren, als lebten wir im neunzehnten Jahrhundert? Man kann nicht die demokratischen Qualitäten eines Parlaments rühmen, das noch nicht einmal ein Initiativrecht hat, also darauf warten muss, ob, dass und wann die europäischen Exekutiven ihm etwas zur Beratung, Anhörung oder Entscheidung vorlegen. Der Vertrag von Lissabon gibt dem Parlament ein bisschen mehr. Was an Defiziten übrig bleibt, ist aber mehr als genug und führt dazu, dass kein einziger namhafter Politiker meint, für dieses Parlament kandidieren zu müssen, solange er im eigenen Land noch etwas zu sagen hat.

Lenz sollte auch hinnehmen, dass der Europäische Gerichtshof (EuGH) unter seiner jahrelangen Mitwirkung an der schleichenden, nicht etwa demokratischen, sondern bürokratischen Ausdehnung der europäischen Zuständigkeiten einen wesentlichen Anteil hat. Der effet utile war ihm ständig wichtiger als die klare Abgrenzung der Fähigkeiten. Lenz treibt die Kritik am Urteil des Bundesverfassungsgerichts auf die Spitze, wenn er meint, es relativiere die öffentliche parlamentarische Auseinandersetzung durch die geheime Beratung des Gerichts, es wolle ,eine justizgeprägte Demokratie" an die Stelle der ,parlamentsgeprägten parlamentarischen Demokratie" setzen und dem Bundestag - oder gar der Kanzlerin, unserer Regierungschefin - vorschreiben, wie sie ihre Integrationsverantwortung wahrzunehmen habe.

Welche „öffentliche Auseinandersetzung" hat es denn mit dem Vertrag von Lissabon gegeben, wie viele Bürger hatten Gelegenheit, ihn zu lesen oder gar zu versuchen, ihn zu verstehen? Warum haben die Regierungen eine solche Angst davor, den Vertrag einem Referendum zu unterwerfen und damit die Verpflichtung auf sich zu nehmen, dem Wahlbürger den genauen Inhalt des kaum lesbaren Vertragswerks zu erklären? Es ist das unbestreitbare Verdienst der Beschwerdeführer und des Verfassungsgerichts, dass es eine öffentliche Diskussion über den Vertrag überhaupt erst gegeben hat, wenigstens ansatzweise. Und wenn das Gericht dafür sorgt, dass die Bundestagsmehrheit und die Bundesregierung das Grundgesetz beachten müssen, dann ist das keine ,justizgeprägte Demokratie", sondern der dringend notwendige Schutz der Verfassung und der in ihr garantierten Rechte der Bürger vor den Leichtfertigkeiten und dem effet utile der jeweiligen Mehrheit.

Natürlich hat das Bundesverfassungsgericht - entgegen den Wünschen des EuGH das unentziehbare Recht und die Pflicht, darüber zu entscheiden, ob sich ein europäi- 
scher Rechtsakt auf den vom Bundestag ratifizierten Vertrag stützt oder keine europarechtliche Grundlage hat. Europa kann nicht mehr Rechte haben, als ihm übertragen wurden. Und natürlich hat das Bundesverfassungsgericht das unentziehbare Recht und die Pflicht, darüber zu entscheiden, ob ein europäischer Rechtsakt gegen den Artikel 1 unseres Grundgesetzes verstößt. Denn da das Grundrecht der Menschenwürde nicht der Verfügungsgewalt des Bundestags unterliegt, kann er es auch nicht irgendeiner europäischen Institution zur Ausgestaltung und Entscheidung übertragen.

Genau an diesem Punkt setzt die grundsätzliche Kritik an den Darlegungen von Lenz an. Es geht nicht darum, etwa einen nationalistischen Nationalstaat als unabänderlich festzuschreiben, sondern darum, dass Europa nach Art und Inhalt demokratisch geschaffen wird. Wir wollen nicht, dass die Regierungen ohne ausdrückliche parlamentarische Entscheidung die europäischen Zuständigkeiten kraft der ihnen innewohnenden exekutiven Weisheit immer weiter ausdehnen. Es stimmt auch nicht, dass eine Regierung „handlungsunfähig" wird, wenn sie an die Entscheidungen des Parlaments gebunden ist. Ich dachte, dass eine parlamentarische Regierung immer an die Entscheidungen des Parlaments gebunden ist. So steht es im Grundgesetz. Man nennt das ,parlamentarische Demokratie".

Lenz beschwört die öffentliche Zustimmung zu Europa, und er ist dabei, sie zu zerstören. Denn diese Zustimmung gilt einem demokratischen Europa. Sie gilt nicht der Übertragung von Hoheitsrechten auf Kommissare und „Hohe Repräsentanten", die wir nicht kennen und die nicht auf Zeit gewählt worden sind. Sie gilt nicht der schleichenden Ausdehnung einer Beamtenhierarchie. Sie gilt nicht einer Polizeibehörde mit diplomatischer Immunität. Sie gilt nicht der zunehmenden Bevormundung der Abgeordneten und Parlamente durch die vereinigten nationalen Regierungen. Natürlich wollen wir ein geeintes Europa. Aber es wird ein Europa der Bürger sein, oder es wird nicht sein.

(Frankfurter Allgemeine Zeitung v. 27.08.2009, S. 34).

Diese so temperamentvolle wie erfahrungsgeprägte Reaktion macht einmal mehr deutlich, dass der stete Rückbezug der europäischen Entwicklung auf die jeweiligen nationalstaatlichen Traditionen und Voraussetzungen zu den unverzichtbaren Elementen des Integrationsprozesses gehören sollte. Dem dadurch entgegenzuwirken, dass man Einrichtungen als sui generis begreift und daraus abzuleiten sucht, sie etablierten, akzeptierten und leistungsfähigen nationalstaatlichen Kontexten vorzuziehen, verdankt sich gleichsam ideologisch überhöhten Positionen und einem überprüfungsbedürftigen Selbstverständnis. Der deutliche Verweis auf den Souverän und seine Vertretung, das Parlament, erscheint gerade nach den europäischen Erfahrungen des vergangenen Jahrhunderts durchaus ange- 
zeigt, ein Verzicht auf entsprechende Akzeptanz- und Legitimationsprozesse hingegen nicht. Im Übrigen vermischen sich in der Diskussion Realphänomene mit disziplinspezifischen Ausdifferenzierungen, persönliche Lebens- und Karrieremuster mit politischen Grundhaltungen und nicht zuletzt ideologischnormative mit professionell-funktionalen Erwägungen. Interessant ist zudem das von einigen der Beteiligten vorgetragene Staatsverständnis, das den historisch gewachsenen europäischen Nationalstaat kurzerhand als ,"̈̈berlebt“ kennzeichnet, ohne zur Kenntnis zu nehmen, dass nahezu alle rechtsstaatlichen und einem Verständnis der Daseinsvorsorge verpflichteten öffentlichen Leistungen über national- oder gliedstaatliche Einrichtungen erbracht werden. Hier geht man den zweiten Schritt vor dem ersten, fehlt jene Distanz, ohne die ein wissenschaftlicher Diskurs, der Praxisrelevanz für sich in Anspruch nimmt, nicht auskommen dürfte, und müsste auch europapolitisch das prinzipiell begrüßenswerte Wollen durch ein operatives Können ergänzt werden.

Im Übrigen umfasst die gegenwärtig erkennbare öffentlich-rechtliche Diskussion weitere für die hier verfolgte Themenstellung entscheidende Fragen. So geht es im Rahmen einer einsetzenden Debatte zum prorium der Rechtswissenschaft erkennbar um disziplinäre Selbstvergewisserung, Veränderungen „von innen“ und Reaktionen ,nach außen“. Die Disziplin sieht sich offenbar unter Rechtfertigungsdruck, sorgt sich um den Stand der theoretischen Reflexion und sucht zwischen förmlicher Gesetzgebung und Anpassung an veränderte Verhältnisse, Befindlichkeiten und Wertungen ihren Platz. Hinzu tritt, dass mit Ausnahme der europäischen Entwicklung die rasant fortschreitende Internationalisierung des Rechts in Deutschland längere Zeit unbeachtet oder aber spezialisierten Einrichtungen überlassen blieb. Erst allmählich scheint das Bewusstsein dafür zu wachsen, dass die vielfältigen grenzüberschreitenden Prozesse entsprechender Regelungssysteme unterhalb des ohnehin kritisch diskutierten Völkerrechts bedürfen. Dass sich damit wiederum auch methodisch beträchtliche Probleme verbinden, ist offenkundig. Kann es deshalb dabei bleiben, die Disziplin als Jurisprudenz und eben nicht als Jurisszienz zu kennzeichnen und die Schnittstelle etwa zu den Sozialwissenschaften darin zu sehen, dass letztere zu erklären, manchmal auch vorherzusagen suchen, während Juristen entscheiden, damit Verantwortung übernehmen und im Rahmen der Rechtsanwendung auch Herrschaft ausüben? Wenn nicht alles täuscht, dürften solch klare Aufgaben- und Funktionszuweisungen angesichts fortschreitender Verflechtungsprozesse kaum durchzuhalten sein, weder analytisch noch empirisch. Die weitere Entwicklung muss gewiss nicht in eine Auflösung disziplinärer oder auch nur fachlicher Schwerpunkte münden, 
legt aber eine erweiterte Kooperation, vielleicht auch arbeitsteilige Verfahren nahe; die kognitiven Anforderungen des Wissenschaftssystem erfordern dies ohnehin, die Praxis erst recht..

\section{Die Bewältigung der Wirtschafts- und Finanzkrise}

Ein zweiter großer Schwerpunkt der gegenwärtigen staats- und europawissenschaftlichen Diskussion richtet sich aus aktuellem Anlass auf die Rolle und Funktion öffentlicher Einrichtungen bei der Bewältigung der Wirtschafts- und Finanzkrise. In den hier unter II. gruppierten Beiträgen (,Der Staat im Zeichen der Krise") geht es um Steuerungsdefizite und Kompetenzvorbehalte, um die Rückbesinnung auf ordnende und gestaltende Politiken, die bislang bei dem Versuch, weltweit agierenden Märkten einen Rahmen zu bieten, als weithin gescheitert gelten. Das behauptete „Politikversagen“ hat auch hier zu einer gleichsam ideologischen Debatte geführt, die über die Wiederherstellung des Primats der Politik den Ruf nach einem handlungsfähigen „starken“ Staat (und arbeitsfähigen Regionalorganisationen) einschließt. In diesem Verständnis habe sich das Markt-Staat-Verhältnis zu Ungunsten des Letzteren verschoben, fänden sich heute Dichotomien, die nicht mehr über Absichtserklärungen und Anreizpolitiken, sondern nur über wirksames politisches Handeln abzubauen sein dürften.

Die G20-Beschlüsse von London und Pittsburgh suchten dieser Ausgangssituation bekanntlich zu entsprechen, zum einen über weitere konjunkturelle Maßnahmen (mit einem Volumen von 5 Billionen Dollar bis Ende 2010), zum anderen über zusätzliche Wirtschafts- und Kapitalhilfen (in Höhe von etwa 1000 Milliarden Dollar) für Schwellen- und Entwicklungsländer über den Internationalen Währungsfonds (IMF). Diese eher situativ begründeten Massnahmen wurden durch ein Bündel strukturell ansetzender Empfehlungen ergänzt, die neben der Bonifrage, einer IWF-Reform und dem Ende für ,Steuerparadiese“ vor allem auf eine globale Finanzmarktreform zielten. So sollen künftig entsprechende Märkte, Akteure und Produkte einer verbesserten Kontrolle und Aufsicht unterworfen werden. Dazu zählen vor allem hochspekulative Fonds, aber auch RatingAgenturen; sie waren vor allem deshalb in die Kritik geraten, weil sie mit wohlwollenden Bewertungen im Prinzip wertloser Papieren zu den Turbulenzen auf den Finanzmärkten entscheidend beitrugen. Schließlich wurden eine dem komplementäre Abschaffung des Bankgeheimnisses ebenso erörtert wie Grundzüge einer ,,neuen Finanzarchitektur“, die Optimisten (oder auch nur unter besonderem Rechtfertigungsdruck stehende Politiker) bereits von Ansätzen zu einer „Weltregierung" sprechen ließ. Offen freilich blieb, wie und durch wen in wel- 
chen Zeiträumen hier neue Formen der Marktregulierung Platz greifen sollten, zumal sich bereits installierte „Frühwarnsysteme“ weder im öffentlichen noch im privaten Bereich als funktionsfähig erwiesen. Global agierende Akteure sahen (und behandelten) den Staat und seine national wie regional agierenden Einrichtungen in einer primär nur ex post agierenden Position, der zudem Kraft wie Kompetenz zur Steuerung weltweiter agierender Märkte fehlte; ordnungspolitische Rahmensetzungen griffen regelmäßig zu kurz, zumal sich die global verfolgten Wachstumsmodelle zunehmend als kaum kompatibel erwiesen. So steht der europäische Kohäsionserfolg, der allerdings mit einer hohen öffentlichen Verschuldung und wachsenden Handelsbilanzdefiziten erkauft wurde, in inzwischen deutlicher Konkurrenz zum asiatischen Modell.

Die gegenwärtigen Auseinandersetzungen um ,feste Regeln“ für die Akteure auf den Finanzmärkten sind inzwischen von deutlicher Unsicherheit geprägt, die auf die jeweils eigene nationale wie sektorale Ausgangssituation zurückzuführen sein dürfte. Zwar wird weiter propagiert, dass man die finanzmarktlich Handelnden ,,vor sich selbst schützen müsse“, doch erweist sich das Instrumentarium von der Zulassung komplexer Produkte über Eigenkapitalvorgaben bis hin zu verstärkten Aufsichtsverfahren als „sperrig“. Auch fehlt es noch immer an einem breiteren Konsens. Selbst die sich in solchen Fragen meist zurückhaltende Kanzlerin spricht angesichts wieder verstärkter Bonizahlungen und verschleppter Reformbemühungen inzwischen von einer ,ziemlich dicken Lippe“ der Bankenvertreter. Dies bezieht sich nicht zuletzt auf den Vorstandsvorsitzenden der Deutschen Bank, Josef Ackermann, dessen Vorstellung von einem EU-weiten Rettungsfonds gegen künftige Krisen eine deutliche Ablehnung erfuhr. So wie sich die Diskussion um Boni, meist Konkretes vermeidend, heute auf eher grundsätzliche Fragen der Einkommenserzielung und Einkommensverwendung (und dabei zu berücksichtigende ethisch-moralische Kategorien) richtet, findet sich auch in der Fondsidee eine Grundhaltung, die härtere Regulierungen abzuwehren sucht. Selbst das derzeit (erneut von Ackermann) vorgetragene Modell, über einen seitens des Bankensektors zu verantwortenden Fonds ein Instrument zur Auflösung der (behaupteten) „Kreditklemme“ zu schaffen, ist nicht ohne Eigennutz, da die Bundesregierung und mit ihr der Steuerzahler im Gegenzug Kreditrisiken in Milliardenhöhe übernehmen soll. So werden konkrete und das heißt operativ greifbare Regelungen immer wieder Kompromissformen zugeführt, die das lösungsbedürftige Problem bestenfalls tangieren; von der diskutierten Einführung einer Börsenumsatzsteuer bis hin zur Verschärfung der Bankenaufsicht ist Ähnliches zu erwarten. Hinzu kommt, dass man sich bei der 
Forderung nach neuen Einrichtungen und Verfahren immer auch der Frage stellen sollte, durch wen diese getragen und wie entsprechende Aufsichts- und Kontrollpolitiken vollzogen werden sollten. Zudem rächt sich die inzwischen beträchtliche und kaum rücksteuerbare Spreizung zwischen den Einkommen im öffentlichen und privaten Bereich. Im Fazit droht ein Zugriffs- und Kompetenzdefizit der öffentlichen Hand, das kurz- wie mittelfristig kaum aufzulösen ist und befürchten lässt, dass die missbräuchlichen Selbststeuerungsprozesse nicht nur im Bankensektor eine Fortsetzung erfahren.

In diesem Kontext erscheint es bemerkenswert, dass seitens der deutschen Wirtschaftswissenschaften, einer einstmals staats- (und sozial-)wissenschaftlichen Disziplin, nur von marginalen Beiträgen zur Diskussion gesprochen werden kann. Weder schien man auch nur annähernd auf die Krisensituation eingerichtet (oder besser: empirisch-analytisch vorbereitet), noch sah man sich in der Lage, den deutlichen und öffentlich karikierten Prognosedefiziten etwaige Erklärungsmuster folgen zu lassen. Auch mit Blick auf Handlungsempfehlungen für die politischen Akteure traten im weiteren Verlauf der Krise erschreckende Defizite zu Tage. Kritiker sprechen inzwischen davon, dass die Praxis den Volks- wie Betriebswirten „weit enteilt“ sei, die Disziplin sich als unzureichend empirisch grundiert und analytisch anspruchslos darstelle, sie zudem international kaum präsent wäre. Zwar beeilten sich zahlreiche „Managementschulen“, ihr herkömmliches Angebot zu erweitern und allzu kruden Marktideologien ein erweitertes Verständnis vom öffentlichen wie privaten Handeln entgegenzusetzen, doch sind über Jahrzehnte entstandene analytische Einseitigkeiten nicht kurzerhand aufzulösen. Von daher kann es nicht verwundern, dass sich die Politik inzwischen kaum noch wirtschaftswissenschaftlichen Sachverstandes bedient, die entsprechenden Einrichtungen zu marginalisieren sucht und es im Ergebnis zu einem direkten Informations- und Interessenaustausch zwischen den politischen und den privatwirtschaftlichen Eliten kommt. Hier sind Nachholbedarfe erkennbar, werden zudem Transparenz- und Öffentlichkeitsdefizite deutlich, die für die Zukunft nicht eben Positives verheißen. Wessen es bedarf, sind über den zu eng definierten wirtschaftswissenschaftlichen Kernbereich hinausgehende Analysen, die institutionelle Konfigurationen, regulative Verfahren und politische Entscheidungsprozesse ebenso einbeziehen wie die Ergebnisse der historischen und vergleichenden Forschung. Die Vergabe des diesjährigen Nobelpreises für Ökonomie an Elinor Ostrom ist ein Beleg dafür, welchen Erkenntnisgewinn eine empirisch-analytisch anspruchsvolle, interdisziplinär lernfähige und international 
präsente Wirtschaftanalyse verspricht. Hier Anschlussfähigkeit zu dokumentieren, dürfte sich lohnen.

\section{Staatlichkeit im Wandel}

Das dieses Heft abschließende Kapitel III (,Die Veränderung der Staatlichkeit im Zeitablauf') sucht die unterschiedlichen in dieser Publikation angesprochenen Aspekte der Staatsdiskussion aufeinander zu beziehen und sie, wo möglich, zusammenzuführen. Das richtet sich zunächst auf jene Veränderungen der Steuerungsdiskussion, denen die meisten entwickelten Gebietskörperschaften im Zeitablauf unterlagen. Der Begriff des state management nimmt, angelsächsischen Traditionen folgend, dabei die hohe Varietät von Zugängen auf und gibt ihr einen Rahmen, ohne sie analytisch zu überhöhen. So öffnet sich der Blick auf die Zyklizität der Diskussion und deren Ergebnisse, werden wechselseitige Lernprozesse deutlich und finden sich angesichts heterogener Kontextbedingungen erwartbare Asymmetrien und Ungleichzeitigkeiten. Zwar gilt auch für die Staatsdiskussion das Diktum innovations travel fast, doch kann (erst recht im internationalen Vergleich) gewiss nicht von einer Pfadabhängigkeit gesprochen werden - weder für die rechtlich-institutionelle Ausgangssituation noch für die organisatorischen, prozessualen oder inhaltlichen Ausprägungen des öffentlichen Handelns. Da wo Konvergenzprozesse erkennbar sind, ergeben sie sich aufgrund struktureller Ähnlichkeiten oder vergleichbarer Probleme, zudem macht sich der Einfluss der Europäischen Union und anderer Formen der Regionalorganisation bemerkbar. Im Ergebnis finden sich triviale Maßstabsvergrößerungen, etwa im Gefolge grenzüberschreitenden Handelns, aber auch substantiellere Überprüfungen etablierter Routinen, die sich dann meist auf eine zu stark ausdifferenzierte Organisation (und damit verbundene Vetopotentiale der beteiligten Akteure), notwendige Verfahrensvereinfachungen (und damit Zeit- und Reagibilitätsgewinn) sowie materielle Konzentrationsprozesse (bis hin zu verstärkter Arbeitsteilung) richten. Als zentrales Defizit erweist sich zunehmend die horizontale wie vertikale Koordination von Entscheidungsträgern, sei es als fehlende Abstimmung auf einer (meist gebietskörperschaftlichen) Ebene oder als „Mehr-EbenenProblem" in Föderalstaaten und anderen mehrstufigen politisch-administrativen (und gesellschaftlichen) Systemen.

Es sind vor allem die staats- und verwaltungswissenschaftich ausgerichteten Sozialwissenschaften, die sich dieser Probleme annehmen, zumal sich angesichts turbulent verändernder Rahmenbedingungen der vor allem seitens der Praxis formulierte Bedarf an Funktionsanalysen beträchtlich erhöht hat. Allerdings sind 
dem entsprechende Arbeiten erkennbar voraussetzungsvoll, methodisch wie empirisch-analytisch. Hier ist Auf- und Nachholbedarf der anwendungsorientierten Forschung erkennbar, reduziert sich der Kreis der wirklich Sachkundigen bislang auf (zu) wenige Wissenschaftler. Ob Föderalismusreform, Interorganisationsanalysen, Trägerschaftsfragen oder die operative Interaktion von europäischer und nationalstaatlicher Ebene, meist findet sich ein nur punktuelles Interesse, das weder der Komplexität des Gegenstandsbereichs noch dem Stand der wissenschaftlichen Erkenntnis gerecht wird. Da zudem eher Fragen der Politikformulierung als des Vollzugs thematisiert werden, bleibt der ,arbeitende Staat ${ }^{\text {“ }}$ (und das schließt das Zusammenwirken in Regionalorganisationen ein) viel zu häufig ohne kompetent-kritische Begleitung. Auch sollte es sich herumgesprochen haben, dass sich Vor- wie Nachteile (und erst recht Wirkungen) institutioneller Arrangements, komplexer Verfahren oder einzelner Politiken erst bei Einbezug von Vollzugsprozessen erkennen lassen. Das aber macht beträchtliche auch empirische Anstrengungen notwendig, denen man sich wiederum gern entzieht. So erklärt sich, dass es im deutschsprachigen Raum bis heute keine den Namen verdienende Wirkungsforschung gibt und sich die Bemühungen meist nur auf den Nachvollzug einzelner materieller Politiken oder Willenbildungsprozesse beschränken. Im Ergebnis wird Erkenntnis- wie Handlungspotential verschenkt, verstärkt man das selbstreferentielle Verhalten der Praxis und isoliert sich gegenüber Zielgruppen, für die man zu arbeiten vorgibt.

$\mathrm{Zu}$ den wenigen zumindest auch praxeologisch ausgerichteten Untersuchungen tritt seit geraumer Zeit eine Forschungsrichtung, die sich auf der analytischen Makroebene der Frage nach der Reformfähigkeit demokratischer politischer Systeme zuwendet. Hier ist man erkennbar darum bemüht, gleichsam ,systemische“ Verwerfungen der repräsentativen Demokratie, der parlamentarischen Entwicklung, oder des intermediärer Selbstverständnisses nicht nur als solche zu konstatieren, sondern sie einer gleichsam demokratiepolitischen Reformagenda zu unterwerfen. „Demokratisierung der Demokratie“ lautet die hierfür vorgestellte Chiffre, die Aufmerksamkeit schon insofern verdient, als es sich nicht um allfällige (und nicht selten wohlfeile) Absichtserklärungen handelt, sondern um die Verbindung (und produktive Konfrontation) normativer Agenden mit rechtlichen, institutionellen, verfahrensbezogenen und, nicht zuletzt, personellen Voraussetzungen. Sollte es zudem gelingen, demokratiepolitische Reformagenden, die sich ihrer gesellschaftlichen und kulturellen Basen bewusst sind, mit den angesprochenen (auf der Meso- und Mikroebene angelegten) Forschungen zum „arbeitenden Staat“ zu verbinden, wäre ein Weg eröffnet, allzu reaktive und 
selbstbezogene staats- wie politikwissenschaftliche Analysen zu überwinden, vielleicht sogar eine auch nach außen gerichtete Akteursrolle von Wissenschaft neu zu legitimieren.

Bevor die nachfolgenden Beiträge die angesprochenen Zugänge ausfüllen und ihnen ihre je spezifische Ausrichtung geben, sei abschließend mit Bedauern vermerkt, dass vier Autoren krankheitsbedingt die engen Publikationsfristen der ZSE nicht einhalten konnten. Die Beiträge von Werner Abelshauser, Paul Kirchhof, Christoph Möllers und Paul Nolte erscheinen deshalb gemeinsam mit einer Zusammenfassung von Horst Dreier, Friedrich Wilhelm Graf und Joachim Jens Hesse in Heft 1/2010 der ZSE. Mit dem Erscheinen dieses Heftes (Ende Februar 2010) verbindet sich dann auch eine gesonderte Buchpublikation aller Beiträge. Herausgeber wie Autoren hoffen, dass mit dieser Publikation die Varietät der staatswissenschaftlichen Diskussion eine adäquate Abbildung erfährt und in einer fragmentierten wie in Teilen polarisierten Diskussionslandschaft weitere Zugänge und Ligaturen erlaubt.

Joachim Jens Hesse 